\title{
METODOLOGÍA DE CONEXIÓN UTILIZANDO NEUROSKY MINDWAVE MW003 CON MATLAB
}

\author{
Connection methodology using \\ Neurosky Mindwave MW003 with MATLAB
}

\section{EPISTEMUS}

ISSN: 2007-8196 (electrónico)

ISSN: 2007-4530 (impresa)

\section{Bryan Quino Ortiz ${ }^{1}$}

José de Jesús Moreno Vázquez ${ }^{2}$

Aldo Rafael Sartorius Castellanos 3

Antonia Zamudio Radilla 4

Marcia Lorena Hernández Nieto ${ }^{5}$

Recibido: 12 de septiembre de 2019 ,

Aceptado: 30 de noviembre del 2019

Autor de Correspondencia:

Bryan Quino Ortiz

Correo:bryanquino97@Hotmail.com

\section{Resumen}

En la actualidad el ímpetu por comprender el funcionamiento del encéfalo ha motivado a compañías como Neurosky en crear e innovar diademas para la obtención de señales encefalográfícas de bajo costo y gran exactitud, enfocadas a la venta para todo tipo de usuario. En el presente trabajo se mostrará la metodología de conexión de la diadema Neurosky MindWave MW003 efectuando el proceso de recepción, envío y configuración inalámbrica (Bluetooth) con el computador, haciendo uso de la librería Thinkgear.h impartida por la empresa Neurosky, realizando un explicación breve y concisa para el uso del dispositivo, estableciendo las características, métodos de operación y funciones principales para su conexión, utilizando la herramienta MATLAB R2015B, el proceso se describe sistemáticamente enfocándose a usuarios inexpertos en la resolución de sus dudas, así mismo contribuir al usuario experimentado en lenguajes de alto nivel en la creación de nuevas aplicaciones.

Palabras clave: MindWave, MATLAB, thinkgear.dll, TG_DATA, Baudrate.

\section{Abstract}

Actually the momentum to understand the operation of the encephalon has motivated the companies like Neurosky in create and innovate headsets to get encephalographic signs of low cost and great exactitude, focused on the sale for all kind of users. In the present paper, the connection methodology will be shown of the Neurosky MindWave MW003 headset the reception process, send and wireless configuration (Bluetooth) in the computer, making use of the Thinkgear.h library, imparted by Neurosky company, making a brief and concise explication for the device use, settings the characteristics, operation methods and principal functions for its connection, using MATLAB R2015B tool, the process describe systematically focused to inexpert users in the resolution of them doubts, likewise contribute to the experiment users in high level languages in the creation of new applications.

Keywords: MindWave, MATLAB, thinkgear.dII, TG_DATA, Baudrate.
1 Tecnológico Nacional De México - Instituto Tecnológico De Minatitlán, Correo: bryanquino97@Hotmail.com 2 Tecnológico Nacional De México - Instituto Tecnológico De Minatitlán, Correo: Jjmv@ltmina.edu.mx

3 Tecnológico Nacional De México - Instituto Tecnológico De Minatitlán, Correo: Aldo_sartorius@Yahoo.com.mx

4 Tecnológico Nacional De México - Instituto Tecnológico De Minatitlán, Correo: Azamudior@Hotmail.com

5 Tecnológico Nacional De México - Instituto Tecnológico De Minatitlán, Correo: Mlhernandez@ltmina.edu.mx 


\section{INTRODUCCIÓN}

En la actualidad las actividades cerebrales han llamado la atención de personas enfocadas en el área de investigación, múltiples empresas comprometidas con el avance científico han fabricado dispositivos capaces de sensar señales encefalográficas (señales emitidas por el encéfalo), tal como lo presenta Kavita V. Singala [1] enfocando su equipo en áreas de desarrollo y entretenimiento, Neurosky es una empresa líder en la venta de diademas a un bajo costo fabricando su línea Mindwave, manteniendo la calidad y precisión en comparación con diversas diademas en el mercado que resultan más precisas y costosas, siendo un impedimento para la mayoría de usuarios.

En el presente trabajo se efectuará sistemáticamente el proceso necesario en la conexión de la diadema Neurosky Mindwave MW003 con cualquier computadora utilizando la comunicación vía bluetooth, tal como lo presenta Wojciech SALABUN [2]. Para ello se hará uso de la librería Thinkgear.h encontrándose en la página de Neurosky para versiones de 64 bits y 32 bits. El enfoque de este trabajo se direcciona al usuario desarrollador, permitiendo utilizar los avanzados comandos matemáticos que consta la herramienta MATLAB R2015B tal como lo presenta K.A.A. Rahman [3], pretendiendo aclarar la conexión de la diadema sintetizando la información de fuentes diversas que resultan ser complejas para personas con conocimientos en programación desalentando la motivación por falta de información concisa.

\section{COLOCACION Y ASPECTO FISICO DE LA DIADEMA NEUROSKY MINDWAVE MW003}

La diadema es un terminal que detecta las señales encefalográficas evitando el ruido ambiental por medio de un algoritmo denominado "eSense" implementado en el chip Thinkgear tal como lo presenta Leonardo Espinoza [4], el cual consta de 7 componentes principales (Figura 1).

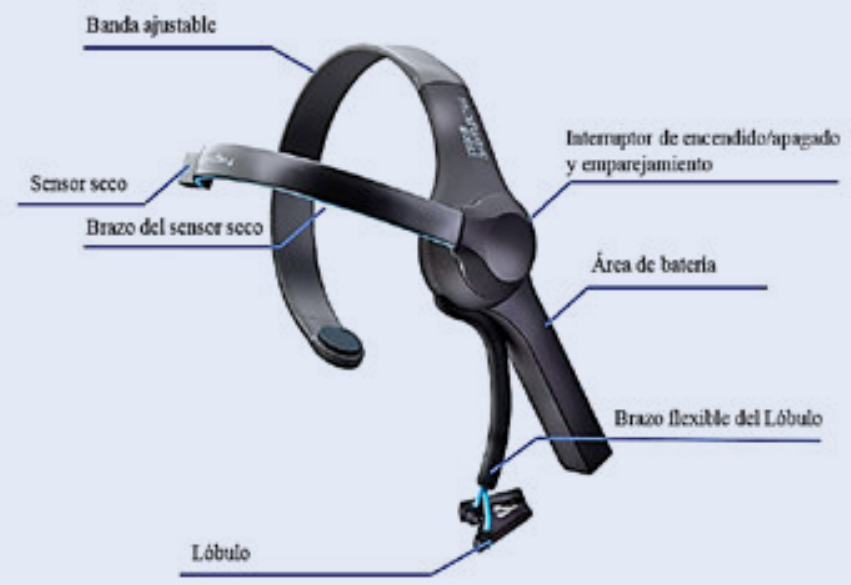

Figura 1. Diseño y componentes principales de Neurosky MindWave MW003.

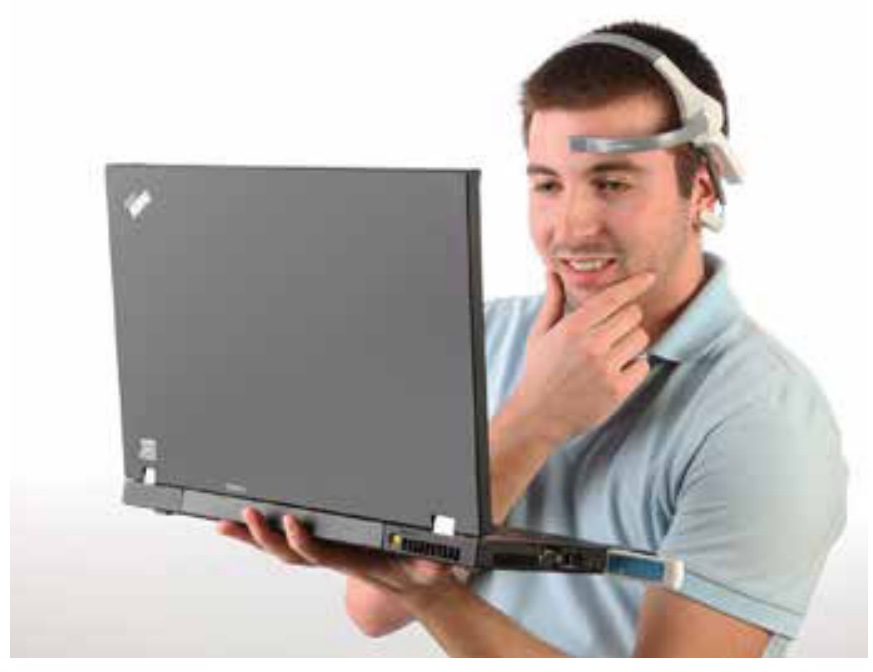

El manual de usuario detalla específicamente la función de cada componente, antes de ser usado se recomienda inspeccionar las instrucciones, tal como lo muestra Pradnya Patil y Dimple Chaudhari [5], comprendiendo el funcionamiento, procedimiento y colocación de los componentes en la toma de lecturas (Tabla 1).

Tabla 1. Especificaciones de los componentes físicos del MindWave MW003.

\section{Componente $\quad$ Descripción/Funcionamiento}

Elemento primario de medición,

Sensor Seco sensa la presencia de voltajes en el rango de los generadas por él encéfalo.

Brazo de sensor

Constituido de un material rígido, seco

Banda ajustable funciona como soporte del sensor seco posicionándolo a la distancia especificada.

Elemento flexible moldeable a la cabeza del usuario, funciona como soporte de la diadema ante presencia de movimientos bruscos.

Interruptor de encendido/ apagado/ emparejamiento

Componente que permite el encendido y apagado de la diadema, como función secundaria sincroniza la comunicación Bluetooth con el equipo (computador).

Área de batería

Sección para el ingreso de la batería, el equipo hace uso de baterías AAA.

Brazo flexible del Elemento maleable, funciona como lóbulo soporte y protección del lóbulo.

Componente aislador, permite Lóbulo retraer el ruido ambiental de la señal medida por el sensor seco. 


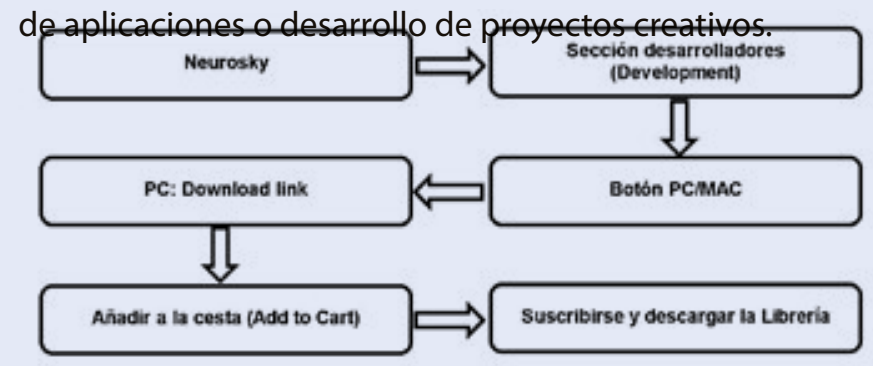

Figura 5. Proceso de descarga de la librería thinkgear.dll.

\section{LIBRERIA THINGEAR.H}

La librería está constituida por funciones que se deberán de llamar de forma sistematizada para emparejar el dispositivo con el equipo (Figure 6). Cada segmento de código contiene comentarios útiles para los usuarios que necesiten modificar parámetros en la implementación de nuevas aplicaciones.

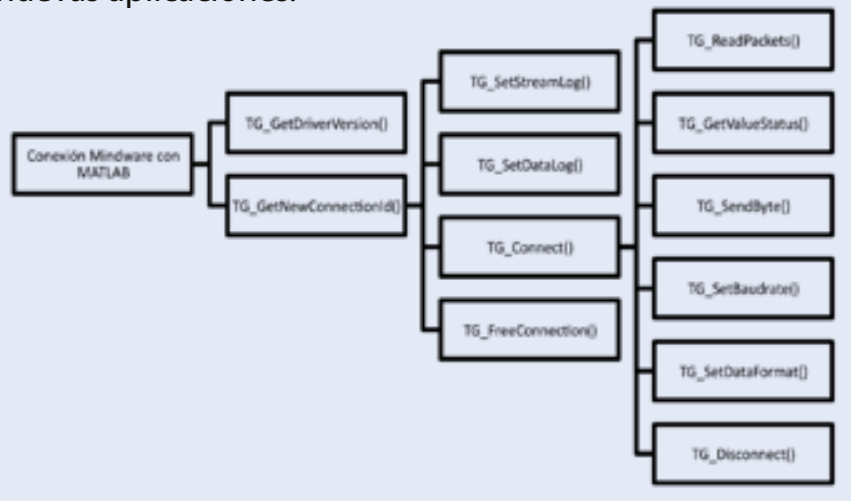

Figure 6. Funciones y Subfunciones configurables dentro de la librería Thinkgear.h.

El Mindwave MW003 maneja 6 tasas de Baudios configurables por el usuario, la tasa de Baudios se debe ingresar considerando las constantes que maneja la librería (Tabla 2). Se recomienda para la comunicación con el computador trabajar a una tasa de Baudios de 57600,

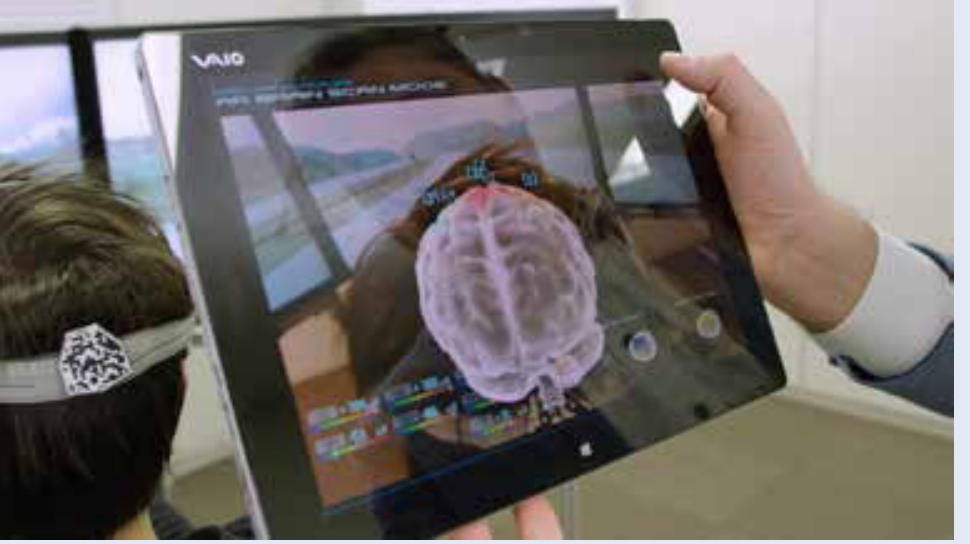

permitiendo que la diadema envié la mayor cantidad de paquetes de datos, una tasa de baudios menor propiciará perdida de información. El chip Thinkgear internamente procesa una cantidad de paquetes de datos establecida por su frecuencia de operación, el receptor del equipo (computador) se establecerá a la misma tasa configurada por la diadema evitando obtener problemas en la recepción de los paquetes de datos.

Tabla 2. Relación de tasa de Baudios y lista de identificadores reconocidos.

\begin{tabular}{|c|c|}
\hline $\begin{array}{c}\text { Identificadores para tasas de } \\
\text { Baudios }\end{array}$ & Baudrate \\
\hline TG_BAUD_1200 & 1200 \\
\hline TG_BAUD_2400 & 2400 \\
\hline TG_BAUD_4800 & 4800 \\
\hline TG_BAUD_9600 & 9600 \\
\hline TG_BAUD_57600 & 57600 \\
\hline TG_BAUD_115200 & 115200 \\
\hline
\end{tabular}

La función TG_GetValueStatus y TG_GetValue hacen uso de la constante TG_DATA, la compañía proporciona un conjunto de constantes capaces de ser procesadas por el chip Thinkgear, referenciando el tipo de señal que se desea recibir por la diadema (Tabla 3). La codificación deberá de llamar a la función TG_GetValueStatus y TG_ GetValue estableciendo una sola constante, de lo contrario se generará un error en el ingreso de datos con la librería. El equipo realiza mediciones de la Señal Raw (señal emitida por el encéfalo sin procesar), amplitud de la señal Alpha baja, Alpha alta, Beta baja, Beta alta, Delta, Gamma baja, Gamma alta y Theta aplicando técnicas de filtrados a un conjunto de muestras tal como lo presenta YU-CHE CHENG [6]. El tiempo de envió de cada paquete de datos es aproximadamente un segundo.

Tabla 3. Identificadores y valores propios para el tipo de señal.

\begin{tabular}{|c|c|}
\hline $\begin{array}{c}\text { Identificadores de datos } \\
\text { procesados }\end{array}$ & Valor \\
\hline TG_DATA_RAW & 4 \\
\hline TG_DATA_DELTA & 5 \\
\hline TG_DATA_THETA & 6 \\
\hline TG_DATA_ALPHA1 & 7 \\
\hline TG_DATA_ALPHA2 & 8 \\
\hline TG_DATA_BETA1 & 9 \\
\hline TG_DATA_BETA2 & 10 \\
\hline TG_DATA_GAMMA1 & 11 \\
\hline
\end{tabular}


El proceso de comunicación establece el uso de 9 funciones necesarias (Tabla 4), la señal procesada por el chip Thinkgear será recibida porTG_GetValue, al finalizar la transferencia de la diadema con el equipo se deberá desconectar por medio de la función TG_FreeConnection liberando el espacio en memoria de la conexión ID abierta por el puerto situando el estado de la diadema en espera, este proceso permite realizar una nueva conexión sin generar errores en la comunicación con MATLAB. La implementación sistematizada de las funciones permite desarrollar programas en otro tipo de lenguaje de alto nivel conociendo la aplicación de sus parámetros, configurando correctamente la librería. Es posible configurar una mayor cantidad de funciones dentro de la librería, sin embargo, no son necesarias en el conexionado de una recepción de datos básica, se recomienda conocer el proceso de envió de datos antes de configurar las funciones no abarcadas en este artículo.

Tabla 4. Lista de funciones principales, orden de ejecución y especificaciones.

\begin{tabular}{|c|c|c|}
\hline $\begin{array}{l}\text { Orden de } \\
\text { configuración }\end{array}$ & Función & Especificación \\
\hline 1 & TG_GetDriverVersion & $\begin{array}{l}\text { Obtiene la versión del driver } \\
\text { enviado por la diadema }\end{array}$ \\
\hline 2 & $\frac{\text { TG_}_{-}}{\text {GetNewConnectionld }}$ & $\begin{array}{l}\text { Retorna un valor entero (Int) del } \\
\text { ID de conexión que se asignó } \\
\text { recientemente. }\end{array}$ \\
\hline 3 & TG_SetStreamLog & $\begin{array}{l}\text { Por medio de la dirección ID, } \\
\text { lee el flujo de bytes dados por } \\
\text { la entrada serial. }\end{array}$ \\
\hline 4 & TG_SetDataLog & $\begin{array}{l}\text { Utiliza la dirección ID } \\
\text { obtenida por la función } \\
\text { TG_GetNewConnecion Id, } \\
\text { depurando problemas. }\end{array}$ \\
\hline 5 & TG_Connect & $\begin{array}{l}\text { Genera una comunicación del } \\
\text { chip Thinkgear por medio de } \\
\text { un puerto COM. }\end{array}$ \\
\hline 6 & TG_ReadPackets & $\begin{array}{l}\text { Lee un paquete de datos y } \\
\text { verifica el arreglo enviado } \\
\text { verificando que sea correcto. }\end{array}$ \\
\hline 7 & TG_GetValueStatus & $\begin{array}{l}\text { Modifica el estado de su valor } \\
\text { si el paquete de datos ha sido } \\
\text { inválido o valido. }\end{array}$ \\
\hline 8 & TG_GetValue & $\begin{array}{l}\text { Guarda el valor procesado por } \\
\text { el chip Thinkgear, enviándolo } \\
\text { por Bluetooth al equipo. }\end{array}$ \\
\hline 9 & TG_FreeConnection & $\begin{array}{l}\text { Libera la memoria asociada con } \\
\text { la conexión del chip ThinkGear } \\
\text { en el computador. }\end{array}$ \\
\hline
\end{tabular}

\section{CONEXIÓN DEL MINDWAVE Y CODIFICACION EN MATLAB}

La codificación en Matlab como en cualquier otro lenguaje de alto nivel deberá seguir el planteamiento mostrado en la (Figura 7), para no provocar errores en la comunicación. 


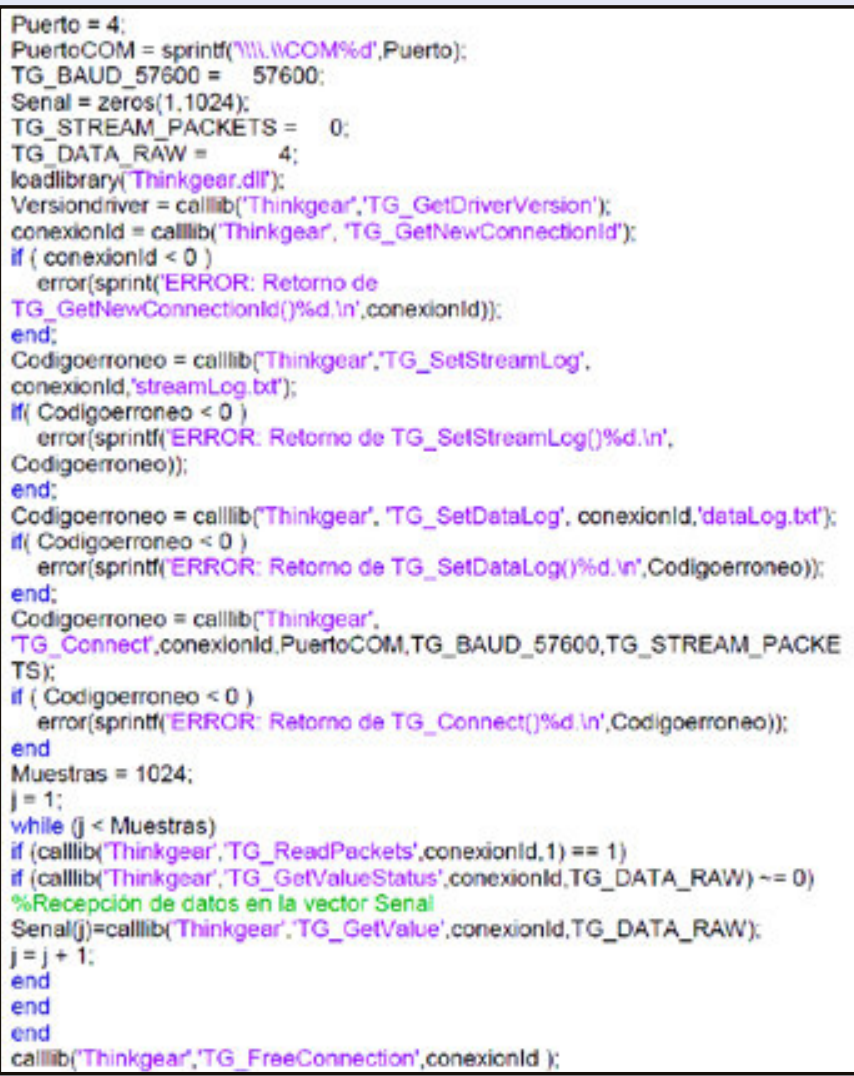

\section{Figura 7. Codificación en Matlab realizada para conexión de Neurosky MindWave MW003.}

La diadema enlaza la comunicación por medio del puerto COM, el código realizado por el usuario no debe provocar errores en la ejecución del archivo.m, en caso contrario la comunicación del Headset (MindWave MW003) se mantendrá abierta generando un error en la ejecución del código al tratar de ejecutarlo nuevamente. Se recomienda resetear el dispositivo generando un nueva conexión, se deberán de ejecutar nuevamente los pasos anteriores con los softwares MindWave Mobile Tutorial y ThinkGear Connector Preferences verificando el puerto de comunicación (COM) enlazado con la diadema. El MindWave envía 512 muestras por segundo haciendo uso de la señal RAW (señal sin procesar), por consecuente, dependiendo del tipo de señal configurada en la variable TG_DATA_RAW será el número de muestras obtenidas tal como lo presenta Bryan Q, Dr. José de Jesús M, Dr. Aldo Rafael S, MDU. Antonia Z and M.C. Marcia L [7]. Una excesiva cantidad de muestras haciendo uso de los filtros generará un tiempo elevado aproximadamente de 18 minutos por sesión, ocupando 1024 muestras, mientras que empleando la señal RAW sería alrededor de 2 segundos por sesión. EI comando callib permite llamar a la librería ubicada dentro de la misma capeta que el archivo.m, en caso contrario no se podrá hacer uso de la librería al ejecutar el código, enviando un mensaje de error al ejecutar el comando loadlibrary().
Antes de ejecutar el código en MATLAB se debe ingresar el comando mex -setup, permitiendo compilar códigos en lenguaje $C$ y en FOLTRAN, se requiere seleccionar mex -setup $\mathrm{C}++$, de lo contrario no será posible compilar el archivo.m que contiene la API (Interfaz de Programación de Aplicaciones), este proceso es obligatorio al utilizar cualquiera de las dos librerías para sistemas de 32 bits y 64 bits, empleando cualquier versión de MATLAB.

\section{CONCLUSION}

En el presente trabajo se procedimiento de forma clara, breve y concisa la metodología de conexionado utilizando el Headset Neurosky MindWave MW003, aplicando la comunicación Bluetooth interactuando con el software MindWave Mobile Tutorial hasta la adquisición de datos con MATLAB R2015B, el procedimiento se llevó a cabo con comandos repetitivos y una codificación pequeña mostrando facilidad en el conexionado haciendo uso de un lenguaje de alto nivel (lenguaje $\mathrm{m}$ ), potenciando los comandos que MATLAB ofrece, cumpliendo el objetivo direccionado a facilitar el entendimiento brindando una guía al usuario, experimentado y no experimentado en el área.

\section{BIBLIOGRAFIA}

[1] K.V. Singala and K.R.Trivedi, "Connection Setup of Openvibe Tool with EEG Headset, Parsing and Processing of EEG signals", International Conference on Communication and Signal Processing (ICCSP), pp.902-906, Abril 2016

[2] W. SALABUN, "Processing and spectral analysis of the raw EEG signal from the MindWave", PRZEGLAD ELEKTROTECHNICZNY, Vol. 2. West Pomeranian University of Technology, Szczecin, pp.68-73, Febrero 2014.

[3] K.A.A. Rahman, B.S.K.K. Ibrahim, M.S. Huq, N.H.M. Nasir M.K.I.Ahmad and F.Sherwani. "Graphics user interface Controlled via brainwave signals for paraplegic rehabilitation", ARPNjournals, Malaysa, 2013

[4] L. Espinosa, "Electroencefalografía Inalámbrica: Una Mirada actual y propuesta de Sistema portátil", Universidad Técnica Federico Santa María, Chile, 2016

[5] P. Patil and D. Chaudhari, "NeuroSky MindWave BCI System: To Save Lives during Transportation", International Journal of Science and Research (IJSR), Vol. 5, pp. 1952-1956, Enero 2016

[6] Y.Cheng, "A hybrid brain-computer interface for intelligent prosthetics",M.C Tesis, Texas A\&M University, Texas, 2014.

[7] Bryan Q, Dr. José de Jesús M, Dr. Aldo Rafael S, MDU. Antonia $Z$ and M.C. Marcia L, "Procesamiento y análisis de señales encefalográficas con Matlab y Neurosky MindWave", Vol. 10, Academia Journals Tabasco 2018, 14-16 de Marzo de 2018.

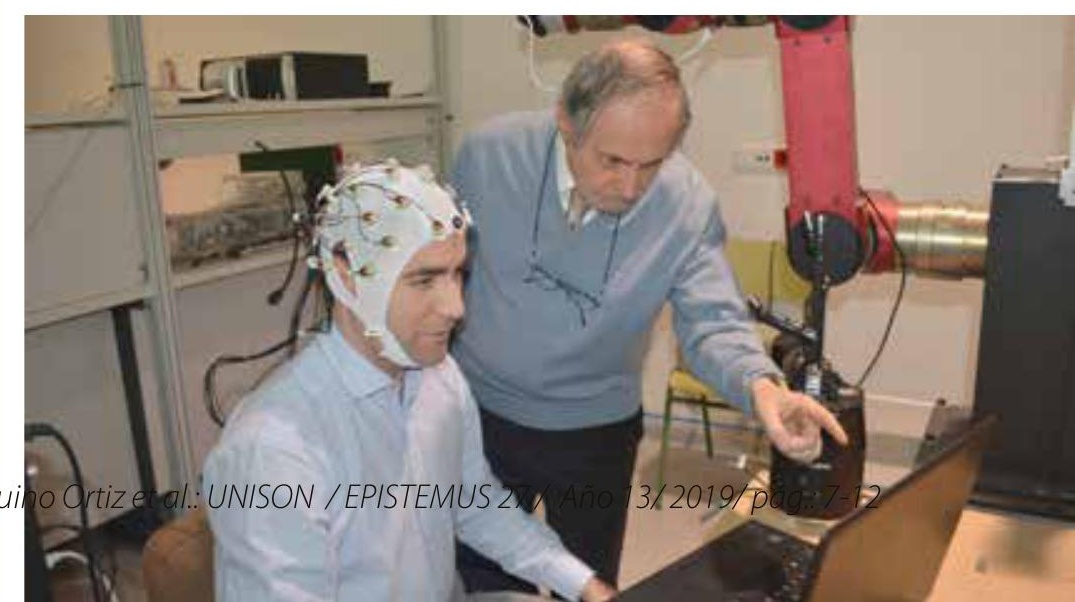

